\title{
METRIC ENTROPY OF HOMOGENEOUS SPACES
}

\author{
STANISEAW J. SZAREK \\ Department of Mathematics, Case Western Reserve University \\ Cleveland, Ohio 44106-7058, U.S.A. \\ E-mail:sjs13@cwru.edu \\ and \\ Equipe d'Analyse, Université Paris VI, Tour 46-00, BP 186 \\ 4, Place Jussieu, 75252 Paris, France
}

\begin{abstract}
For a precompact subset $K$ of a metric space and $\varepsilon>0$, the covering number $N(K, \varepsilon)$ is defined as the smallest number of balls of radius $\varepsilon$ whose union covers $K$. Knowledge of the metric entropy, i.e., the asymptotic behaviour of covering numbers for (families of) metric spaces is important in many areas of mathematics (geometry, functional analysis, probability, coding theory, to name a few). In this paper we give asymptotically correct estimates for covering numbers for a large class of homogeneous spaces of unitary (or orthogonal) groups with respect to some natural metrics, most notably the one induced by the operator norm. This generalizes the author's earlier results concerning covering numbers of Grassmann manifolds; the generalization is motivated by applications to noncommutative probability and operator algebras. The argument uses a characterization of geodesics in $U(n)$ (or $S O(m)$ ) for a class of non-Riemannian Finsler metric structures.
\end{abstract}

1. Introduction. If $(M, \rho)$ is a metric space, $K \subset M$ a precompact subset and $\varepsilon>0$, the covering number $N(K, \varepsilon)=N(K, \rho, \varepsilon)$ is defined as the smallest number of balls of radius $\varepsilon$ whose union covers $K$. If $K$ is a ball of radius $R$ in a normed space of real dimension $d$, it is easily shown (by a standard volume comparison argument, see [23]) that, for any $\varepsilon \in(0, R]$,

$$
(R / \varepsilon)^{d} \leq N(K, \varepsilon) \leq(1+2 R / \varepsilon)^{d} .
$$

The lower and the upper estimate in (1) differ roughly by a factor of $2^{d}$, and for many applications such an accuracy is sufficient. On the other hand, determining more precise asymptotics for covering numbers and their "cousins", packing numbers (see section 2),

1991 Mathematics Subject Classification: Primary 52C17, 53C30, 46L50; Secondary 53C60, $53 \mathrm{C} 22$.

Research partially supported by grants from the National Science Foundation.

The paper is in final form and no version of it will be published elsewhere. 
e.g. for Euclidean balls is a nontrivial proposition (and a major industry). In this paper we attempt to obtain estimates of type (1) for homogeneous spaces of the orthogonal group $S O(n)$ or the unitary group $U(n)$ (like e.g. the Grassmann manifold $G_{n, k}$ or flag manifolds, equipped with some natural metric; we admit metrics induced by unitary ideal norms of matrices, most notably the operator norm). A typical result will be: if $M$ is a "nice" homogeneous space of $S O(n)$ or $U(n)$ and $\varepsilon \in(0, \theta(M)]$ (where $\theta(M)$ is some computable "characteristic" of $M$, in the "regular" cases $\theta(M) \approx \operatorname{diam} M)$, then

$$
(c \operatorname{diam} M / \varepsilon)^{d} \leq N(M, \varepsilon) \leq(C \operatorname{diam} M / \varepsilon)^{d},
$$

where $d$ is the (real) dimension of $M$ and $c$ and $C$ are constants independent of $\varepsilon$ and (largely) of $M$. Of course universality of the constants in question is the crucial point.

We note in passing that (again, by a standard "volume comparison" argument) (2) is equivalent to the (normalized) Haar measure of a ball of radius $\varepsilon$ being between $\left(c_{1} \varepsilon / \operatorname{diam} M\right)^{\operatorname{dim} M}$ and $\left(C_{1} \varepsilon / \operatorname{diam} M\right)^{\operatorname{dim} M}$, where $c_{1}, C_{1}>0$ are constants depending only on $c, C$. We also point out that the underlying metric being typically nonRiemannian, the methods of Riemannian geometry do not apply directly.

This paper is an elaboration of the note [17] by the author, where (2) was proved in the special case of $M$ being $S O(n), U(n)$ or a Grassmann manifold $G_{n, k}$. (Another argument for that was given later in [13].) The original motivation and application was the finite-dimensional basis problem; more precisely, (2) was used in the proof of (Theorem 1.1 in [18]):

There is a constant $c>0$ such that, for every positive integer $n$, there exists an $n$-dimensional normed space $B$ such that, for every projection $P$ on $B$ satisfying, say, $0.01 n \leq \operatorname{rank} P \leq 0.99 n$, we have $\|P: B \rightarrow B\|>c \sqrt{n}$.

The results of [17] were subsequently applied to other problems in convexity, local theory of Banach spaces, operator theory, noncommutative probability and operator algebras (cf. e.g. [7, 8, 12, 19, 20, 25, 26]). It turned out recently (see [25]) that some questions from the last two fields lead naturally to queries about validity of estimates of type (2) in settings more general than that of the paper [17] (which, additionally, had a rather limited circulation). It is the purpose of this paper to provide a reasonably general answer to such questions; describing asymptotics for completely general homogeneous spaces of $S O(m)$ or $U(n)$ is, in all likelihood, hopeless. We cover here a number of special cases, including those that have been explicitly inquired about. We identify (easily computable) invariants relevant to the problem and provide "tricks" that could be potentially useful to handle cases not addressed here. Except for brief comments here and there, we restrict our attention to $M=G / H$, where $G=S O(m)$ or $U(n)$, and $H$ a connected Lie subgroup of $G$; but clearly most of our analysis can be extended to other compact linear Lie groups.

The organization of the paper is as follows.

Section 2 explains notation and presents various preliminary results concerning covering numbers and their relatives, unitarily invariant norms and the exponential map. Most of these are known or probably known. In particular we state there Proposition 6 which asserts that cosets of one parameter semigroups are the geodesics in $U(n)$ (or $S O(m)$ ) endowed with an intrinsic metric induced by a unitarily invariant norm; this result could 
be of independent interest. In section 3 we discuss several simple examples that exemplify possible obstructions to estimates of type (2) and suggest invariants mentioned above. We then use the results of the preceding section to show estimates of type (2) for an abstract class of homogeneous spaces that contains $U(n), S O(n)$ and $G_{n, k}$ (Theorem 8). In section 4 we discuss various possibilities for relaxation of assumptions from section 3 , in particular we cover the special cases motivated by applications. At the end of section 4 we briefly address the issue of extending the results to metrics generated by unitarily invariant norms other than the operator norm.

Acknowledgements. The author would like to express his gratitude to D. Voiculescu, whose encouragement was instrumental both in the inception and the completion of this work. The final part of the research has been performed while the author was in residence at MSRI Berkeley during the spring semester of 1996; thanks are due to the staff of the institute and the organizers of the Convex Geometry semester for their hospitality and support, and to many visitors and members of the institute for contributing in a variety of ways to a stimulating atmosphere there. A preliminary exposition of the results was included in the preprint [21].

2. Notation and preliminaries. We start with several largely trivial remarks clarifying the relationship between covering numbers, packing numbers and their slightly different versions that exist on the market. First, since the centers of balls in the definition of $N(K, \varepsilon)$, as given in the introduction, do not necessarily need to be in $K$, the exact value of $N(K, \cdot)$ may depend on the ambient metric space $(M, \rho)$ containing $K$. Accordingly, it is sometimes more convenient to allow sets of diameter $\leq 2 \varepsilon$ in place of balls of radius $\varepsilon$; call the resulting the quantity $N^{\prime}(K, \varepsilon)$. If the centers of the balls in the definition are required to be in $K$, call the quantity $N^{\prime \prime}(K, \varepsilon)$. Finally, let the packing number $\tilde{N}(K, \varepsilon)$ be defined as the maximal cardinality of an $\varepsilon$-separated (i.e. $\rho\left(x, x^{\prime}\right)>\varepsilon$ if $\left.x \neq x^{\prime}\right)$ set in $K$. The quantities $N, N^{\prime}$ and $\tilde{N}$ are related as follows

$$
N^{\prime}(\cdot, \varepsilon) \leq N(\cdot, \varepsilon) \leq N^{\prime \prime}(\cdot, \varepsilon) \leq \tilde{N}(\cdot, \varepsilon) \leq N^{\prime}(\cdot, \varepsilon / 2) .
$$

Consequently, for our "asymptotic" results the four quantities are essentially interchangeable.

If the metric space $(M, \rho)$ is actually a normed space with a norm $\|\cdot\|$ and unit ball $B$, we may write $N(K,\|\cdot\|, \varepsilon)$ or $N(K, B, \varepsilon)$ instead of $N(K, \varepsilon)$ or $N(K, \rho, \varepsilon)$. The technology for estimating covering/packing numbers of subsets (particularly convex subsets) of normed spaces is quite well-developed and frequently rather sophisticated (see [4], [14]). We quote here a simple well-known result (again, proved by a "volume comparison" argument) that expresses $N(\cdot, \cdot)$ in terms of a "volume ratio", and of which (1) is a special case.

Lemma 1. Let $K, B \subset R^{d}$ with $B$ convex symmetric. Then, for any $\varepsilon>0$,

$$
\left(\frac{1}{\varepsilon}\right)^{d} \frac{\operatorname{vol} K}{\operatorname{vol} B} \leq N(K, B, \varepsilon) \leq N^{\prime \prime}(K, B, \varepsilon) \leq N^{\prime}(K, B, \varepsilon / 2) \leq\left(\frac{2}{\varepsilon}\right)^{d} \frac{\operatorname{vol}(K+\varepsilon / 2 B)}{\operatorname{vol} B}
$$

The next lemma is just an observation which expresses the fact that the covering/packing numbers are invariants of Lipschitz maps. 
Lemma 2. Let $(M, \rho)$ and $\left(M_{1}, \rho_{1}\right)$ be metric spaces, $K \subset M, \Phi: K \rightarrow M_{1}$, and let $L>0$. If $\Phi$ satisfies

$$
\rho_{1}(\Phi(x), \Phi(y)) \leq L \rho(x, y) \text { for } x, y \in K
$$

(i.e. $\Phi$ is a Lipschitz map with constant $L)$, then, for every $\varepsilon>0$,

$$
N^{\prime \prime}\left(\Phi(K), \rho_{1}, L \varepsilon\right) \leq N^{\prime \prime}(K, \rho, \varepsilon) .
$$

Moreover, $N^{\prime \prime}$ can be replaced by $N^{\prime}$ or $\tilde{N}$ and, if $\Phi$ can be extended to a function on $M$ that is still Lipschitz with constant L, also by $N$.

We now turn to our main interest, the unitary group $U(n)$, the (special) orthogonal group $S O(n)$ and their homogeneous spaces. (As $O(n)$ is geometrically a disjoint union of two copies of $S O(n)$, all statements about $S O(n)$ will easily transfer to $O(n)$.) Throughout the paper we will reserve the letter $G$ to denote, depending on the context, $U(n)$ or $S O(n)$. Similarly, we will reserve the letter $\mathcal{G}$ to denote the Lie algebra of $G$, the space $u(n)$ or $s o(n)$ of skew-symmetric matrices. Since $G$ and $\mathcal{G}$ are subsets of $M(n)$ (the algebra of $n \times n$ matrices, real or complex as appropriate), they inherit various metric structures from the latter. In this paper we focus on the one induced by the operator norm (as an operator on the Euclidean space, that is), but will also consider the Schatten $C_{p}$-norms $\|x\|_{p}=\left(\operatorname{tr}|x|^{p}\right)^{1 / p}$ with the operator norm $\|\cdot\|_{o p}=\|\cdot\|_{\infty}$ being the limit case. We will use the same notation $\|x\|_{p}$ for the $\ell_{p}^{n}$-norm on $\mathbf{R}^{n}$ or $\mathbf{C}^{n}$, but this should not lead to confusion. More generally, we will also occasionally look at other unitarily invariant norms (i.e. satisfying $\|x\|=\|u x v\|$ if $x \in M(n)$ and $u, v \in G)$, each necessarily associated with a symmetric norm on $\mathbf{R}^{n}$ (which we will also denote by $\|\cdot\|$ ) via $\|x\|=\left\|\left(s_{k}(x)\right)_{k=1}^{n}\right\|$, where $s_{1}(x), \ldots, s_{n}(x)$ are singular numbers of $x$.

If $\rho$ is a metric on $G$ and $H \subset G$ a closed subgroup, we consider the homogeneous space $M=G / H$ of left cosets of $H$ in $G$ as endowed with the canonical quotient metric $\rho_{M}(E, F)=\inf \{\rho(u, v): u \in E, v \in F\}$. A fundamental example is that of the Grassmann manifold $G_{n, k}$ of $k$-dimensional subspaces of $\mathbf{R}^{n}$ (resp. $\mathbf{C}^{n}$ ): the relevant subgroup $H$ of $S O(n)$ (resp. $U(n)$ ) consists of matrices of the form

$$
\left[\begin{array}{cc}
u_{1} & 0 \\
0 & u_{2}
\end{array}\right]
$$

where $u_{1} \in S O(k)$ and $u_{2} \in S O(n-k)$ (resp. $U(k), U(n-k)$ ) and the identification of cosets of $H$ with the subspaces is via $u H \sim u E_{k}$, where $E_{k}$ is the linear span of the first $k$ vectors of the standard basis of $\mathbf{R}^{n}$ (resp. $\mathbf{C}^{n}$ ).

Among metric structures on $M=U(n)$ (or $S O(n)$, or the homogeneous space), of particular interest to us will be intrinsic metrics induced by (unitarily invariant) norms on $M(n)$ : the distance between $u$ and $v$ is the infimum of lengths of curves in $M$ connecting $u$ and $v$. We observe that the infimum may be taken over absolutely continuous curves $\gamma:[a, b] \rightarrow M$ (the infimum is then achieved, while any rectifiable curve parametrized by arc length is absolutely continuous). Then the length

$$
\ell(\gamma)=\int_{0}^{1}\left\|\gamma^{\prime}(t)\right\| d t
$$


where, if $M=G,\|\cdot\|$ is the norm on $M(n)$ in question. If $M=G / H,\|\cdot\|$ can be interpreted as a quotient norm on the corresponding quotient of the relevant Lie algebra (cf. (9) in section 3 and comments following it). We also point out that all the metrics we consider being bi-invariant, any curve in $M=G / H$ can be lifted (by compactness and elementary properties of the $L_{1}$-norm) to a "transversal" curve in $G$ of the same length. The correct abstract framework for these considerations is that of Finsler geometry (see e.g. [3]), but since the manifolds we consider are canonically embedded in natural normed spaces, we can afford to be more "concrete".

For future reference we point out that the (operator) norm distance and the corresponding intrinsic distance $\rho$ are related via

$$
\|u-v\|=\left|1-e^{i \rho(u, v)}\right| .
$$

This is an immediate consequence of Proposition 6 that follows; however, here we just wish to point out that the two metrics differ by a factor of $\pi / 2$ at the most and this particular fact is implied by the more or less obvious inequalities $\rho(u, v) \geq\|u-v\| \geq\left|1-e^{i \rho(u, v)}\right|$. Since, by definition, the quotient metrics are distances between cosets, the corresponding two metric structures on homogeneous spaces are related in the way analogous to (6). Accordingly, estimates of type (2) will transfer easily from one metric to the other, and the choice of the one to work with will only be a matter of convenience and/or elegance.

Because of the invariance of the metric under the action of $G(=U(n)$ or $S O(n))$, one can give estimates for the covering numbers of $M$ (analogous to those of Lemma 1) in terms of the Haar measures of balls (cf. the comment following (2)). However, since the dependence of the measure of a ball on the radius is much less transparent now than in the "linear" case, such estimates are not necessarily useful. To overcome this difficulty we "linearize" the problem via the exponential map (composed with the quotient map $q: G \rightarrow M$ if necessary) and then use Lemma 2. Since we operate in the "classical" context, the exponential map is the standard one

$$
\exp x=e^{x}=\sum_{k=0}^{\infty} \frac{x^{k}}{k !} \text { for } x \in M(n),
$$

and it will be normally sufficient to consider the restriction of exp to $\mathcal{G}(=u(n)$ or $s o(n))$, the Lie algebra of $G(=U(n)$ or $S O(n))$. In order to be able to apply Lemma 2 we must "understand" the map $\Phi=q \circ \exp$. Specifically, we need to know for which $K \subset \mathcal{G}$ we have $\Phi(K)=M$ (or at least when $\Phi(K)$ is "large") and for which $K$ the restriction $\Phi_{\mid K}$ (resp. $\Phi_{\mid \Phi(K)}^{-1}$ ) is Lipschitz. Concerning the first point, it is well known that, in our context, $\exp (\mathcal{G})=G$. Moreover, we have

Lemma 3. Let $K=\left\{x \in \mathcal{G}:\|x\|_{\infty} \leq \pi\right\}$ be the ball of radius $\pi$ in $\mathcal{G}$ in the operator norm. Then

(a) $\exp (K)=G$,

(b) $\exp$ is one-to-one on the interior of $K$.

The above is a special case of a more general fact for Lie groups, but in the present setting can be seen directly from the fact that every unitary matrix can be diagonalized, with the argument for $S O(n)$ being just slightly more complicated. 
Lemma 3 asserts that $G$ resembles, in a sense, a ball in $\mathcal{G}$. However, for our purposes we need more quantitative information about exp, which we collect in the next lemma.

LEMMA 4. For any unitarily invariant norm and the corresponding metric on $G$ (extrinsic on intrinsic), the map $\exp : \mathcal{G} \rightarrow G$ is a contraction.

On the other hand, let $\|\cdot\|$ be such a unitarily invariant norm and set, for $\theta>0$,

$$
\phi(\theta)=\inf \left\{\left\|e^{x}-e^{y}\right\| /\|x-y\|: x, y \in \mathcal{G}, x \neq y,\|x\|_{\infty} \leq \theta,\|y\|_{\infty} \leq \theta\right\} .
$$

Then $\phi(\theta)>0$ if $\theta<\pi$. Moreover, if $\theta \in[0,2 \pi / 3)$, then

$$
\phi(\theta) \geq \prod_{k=1}^{\infty}\left(1-\left|1-e^{i \theta / 2^{k}}\right|\right) .
$$

In particular $\phi(\theta) \geq 0.4$ if $\theta \leq \pi / 4$.

Proof. The first assertion is classical for the extrinsic (norm) metric and hence follows formally for the intrinsic metric. For the other assertions, we observe first that since the derivative of the exponential map at 0 is the identity,

$$
\lim _{\theta \rightarrow 0^{+}} \phi(\theta)=1 \text {. }
$$

Let $x, y$ be as in the definition of $\phi(\theta)$. We have

$$
\begin{aligned}
e^{x}-e^{y} & =e^{\frac{x}{2}}\left(e^{\frac{x}{2}}-e^{\frac{y}{2}}\right)+\left(e^{\frac{x}{2}}-e^{\frac{y}{2}}\right) e^{\frac{y}{2}} \\
& =2\left(e^{\frac{x}{2}}-e^{\frac{y}{2}}\right)+\left(e^{\frac{x}{2}}-I\right)\left(e^{\frac{x}{2}}-e^{\frac{y}{2}}\right)+\left(e^{\frac{x}{2}}-e^{\frac{y}{2}}\right)\left(e^{\frac{y}{2}}-I\right)
\end{aligned}
$$

and so, by the ideal property of unitarily invariant norms,

$$
\begin{aligned}
\left\|e^{x}-e^{y}\right\| & \geq\left\|e^{\frac{x}{2}}-e^{\frac{y}{2}}\right\|\left(2-\left\|e^{\frac{x}{2}}-I\right\|_{\infty}-\left\|e^{\frac{y}{2}}-I\right\|_{\infty}\right) \\
& \geq \phi(\theta / 2)\|x-y\| \cdot\left(1-\left|1-e^{\frac{i \theta}{2}}\right|\right) .
\end{aligned}
$$

Iterating and using (7) we obtain the third (and hence the last) assertion of the lemma. For the second assertion $(\phi(\theta)>0$ if $\theta<\pi$, not used in the sequel), we just briefly sketch the argument for $G=U(n)$. Let $\theta \in(0, \pi)$ and $\delta>0$. Consider first the case of the operator norm. We need to show that if $A, B$ are Hermitian with spectra contained in $[-\theta, \theta]$ and $\left\|e^{i A}-e^{i B}\right\| \leq \delta$, then $\|A-B\| \leq C(\theta) \delta$, where $C(\theta)$ depends only on $\theta$ (and not on $A, B$ or $n$ ). By [2], Theorem 13.6, the eigenvalues of $A$ and $B$ (multiplicities counted) are, in a certain precise sense, "close", and so, by perturbation, we may assume that they are identical; we may also assume that all those eigenvalues are integral multiples of $\delta$. Let $u \in U(n)$ be such that $B=u A u^{-1}$; we need to show that $\left\|e^{i A} u-u e^{i A}\right\| \leq 4 \delta$ implies $\|A u-u A\| \leq C(\theta) \delta$ and this follows by writing $u$ as a "block matrix" in the spectral subspaces of $A$. For a general unitarily invariant norm we note that the assertion is roughly equivalent to uniform boundedness (with respect to the norm in question and with a bound depending only on $\theta$ ) of the inverse of the derivative of the exponential map. That derivative is, in a proper orthonormal basis, an antisymmetric "Schur multiplier" (see [24], Theorem 2.14.3 and its proof). As a consequence, the inverse is also such a multiplier, and so its norm with respect to the operator norm equals to the norm on the trace class $C_{1}$ (by duality) and dominates the norm with respect to any unitarily invariant norm by interpolation (cf. [23], $§ 28$ or [10]). 
Re marks. (i) In all likelihood, a version of Lemma 4 (and of Lemma 5 that follows) should be known, at least for the operator norm, but we couldn't find a reference. It would be nice to have an elegant proof which gives good constants in the full range of $\theta(\in(0, \pi))$. We point out that the "Schur multiplier" argument indicated above provides a simple "functional calculus" proof (with fairly good constants) in the case of the Hilbert-Schmidt $C_{2}$-norm.

(ii) What is really important in Lemma 3 and the auxiliary results that follow is that the estimates are "dimension free". We could have used cruder, and slightly easier to prove, inequalities (e.g., it would be enough to know that $\phi(\theta)>0$ for some $\theta>0$ ), but we thought it worthwhile to add a few lines here and there to get reasonable, if not optimal, estimates.

Lemma 5. Let $G=U(n)$ (resp. $S O(n))$ and $\rho$ the intrinsic metric on $G$ induced by a unitarily invariant norm $\|\cdot\|$ on $M(n)$. Then, for any $x, y \in \mathcal{G}$,

$$
\rho\left(e^{x+y}, e^{x} e^{y}\right) \leq\|[x, y]\| \text {. }
$$

Pr o of. The argument is similar to, but slightly more complicated than that of the previous lemma. Denote, for $t \geq 0$,

$$
\psi(t)=\max \left\{\rho\left(e^{t(x+y)}, e^{t x} e^{t y}\right), \rho\left(e^{t(x+y)}, e^{t y} e^{t x}\right)\right\} .
$$

Clearly $\psi(0)=0$. Moreover, expanding the exponentials and noting that $\rho(u, v) /\|u-v\|$ $\rightarrow 1$ as $\|u-v\| \rightarrow 0$ (this follows from (6), but can also be seen from the inequalities in the paragraph following (6), which do not depend on Proposition 6) we conclude that

$$
\lim _{t \rightarrow 0} \psi(t) / t^{2}=\|[x, y]\| \text {. }
$$

Now

$$
\begin{aligned}
\rho\left(e^{x+y}, e^{x} e^{y}\right) & \leq \rho\left(e^{x+y}, e^{\frac{x+y}{2}} e^{\frac{x}{2}} e^{\frac{y}{2}}\right)+\rho\left(e^{\frac{x+y}{2}} e^{\frac{x}{2}} e^{\frac{y}{2}}, e^{\frac{x}{2}} e^{\frac{y}{2}} e^{\frac{x}{2}} e^{\frac{y}{2}}\right) \\
& +\rho\left(e^{\frac{x}{2}} e^{\frac{y}{2}} e^{\frac{x}{2}} e^{\frac{y}{2}}, e^{\frac{x}{2}} e^{\frac{x+y}{2}} e^{\frac{y}{2}}\right)+\rho\left(e^{\frac{x}{2}} e^{\frac{x+y}{2}} e^{\frac{y}{2}}, e^{x} e^{y}\right) \\
& =3 \rho\left(e^{\frac{x+y}{2}}, e^{\frac{x}{2}} e^{\frac{y}{2}}\right)+\rho\left(e^{\frac{y}{2}} e^{\frac{x}{2}}, e^{\frac{x+y}{2}}\right) .
\end{aligned}
$$

Hence $\psi(1) \leq 4 \psi\left(\frac{1}{2}\right)$ and, by the same argument, $\psi(t) \leq 4 \psi\left(\frac{t}{2}\right)$ or $\frac{\psi(t)}{t^{2}} \leq \frac{\psi\left(\frac{t}{2}\right)}{\left(\frac{t}{2}\right)^{2}}$ for $t \geq 0$. In combination with (8) this implies the lemma.

Our last auxiliary result involves the non-Riemannian geometry of $G=U(n)$ (or $S O(n))$. It is very well known, in a much more general context, that if $G$ is endowed with a bi-invariant Riemannian structure (which is, in our case, the one induced by the Hilbert-Schmidt $C_{2}$-norm on $M(n)$ ), then the geodesics of $G$ are exactly the cosets of one-parameter subgroups (see [11], p. 148, Ex. 5, 6). However, it is not immediately clear how general is this phenomenon. Since geodesics are normally defined via affine connections, we reiterate here that we employ the "metric" approach in the spirit of classical Finsler geometry: a curve in a manifold $M$ endowed with a metric is a geodesic if it locally realizes the (intrinsic) distance between points as explained in the paragraph containing (5). We have

Proposition 6 ([22]). Let $\|\cdot\|$ be a unitarily invariant norm on $M(n)$ and $\rho$ the induced intrinsic metric on $G(=U(n)$ or $S O(n))$. Then 
(a) cosets of one parametric semigroups (i.e. curves of the form $\gamma(t)=u e^{t x}, u \in G$, $x \in \mathcal{G})$ are geodesics in $(G, \rho)$,

(b) if $\|\cdot\|$ is strictly convex (which happens in particular if $\|\cdot\|=\|\cdot\|_{p}$ for some $p \in(1, \infty))$, then all geodesics are, up to a change of parameter, of the form given in $(a)$ (or arcs of curves of such form),

(c) if, furthermore, the spectrum of $u^{-1} v$ does not contain -1 , the curve of shortest length (geodesic arc) connecting $u$ and $v$ is unique.

Remarks. (i) A unitarily invariant norm on $M(n)$ is strictly convex (i.e. the unit sphere $\{x:\|x\|=1\}$ does not contain a segment) iff the associated symmetric norm on $\mathbf{R}^{n}$ is (this is, e.g., implicit in [10], §II.4 and $\S$ III.3; see also [1] for a "definite" treatment of the $C_{p}$ case). The operator norm and the trace class $C_{1}$-norm are not strictly convex, while the Schatten $C_{p}$-norms for $p \in(1, \infty)$ are strictly convex.

(ii) The proof of Proposition 6 is based on (and in fact very close to) the results on spectral variation of unitary matrices presented in [2], $§ 13,14$.

3. The "1-cocomplemented" subgroups. Let $G=S O(m)$ or $U(n)$ and $\mathcal{G}(=$ so $(n)$ or $u(n))$ the Lie algebra of $G$. Let $H$ be a connected Lie subgroup of $G, \mathcal{H}$ the corresponding Lie subalgebra of $\mathcal{G}$ and $M=G / H$. In this section we concentrate on the case when $G$ and $M$ are endowed with metric structures induced by the operator norm, we will denote the respective metrics by $\rho$ and $\rho_{M}$ (for technical purposes, we may use other unitary ideal norms, though). The purpose of this section is to prove, in the above context, an estimate of type (2) for an abstract class of homogeneous spaces that contains $U(n), S O(n)$ and the Grassmannians $G_{n, k}$. The argument will depend on a careful, but elementary analysis of the exponential map exp : $\mathcal{G} \rightarrow G$ and maps obtained from it; as an illustration we point out here that the following result from [17] is an immediate consequence of the results from the preceding two sections.

THEOREM 7. If $G=S O(n)$ or $U(n)$ (endowed with the operator norm or the induced intrinsic metric $\rho)$ and $\varepsilon \in(0,2]$, then

$$
(c / \varepsilon)^{d} \leq N(G, \varepsilon) \leq(C / \varepsilon)^{d},
$$

where $d$ is the (real) dimension of $G$ and $c$ and $C$ are universal numerical constants.

P r o o f. By Lemma 3 and the first assertion of Lemma 4, exp is a contractive surjective map from the (closed) ball of radius $\pi$ in $\mathcal{G}$ to $G$. Consequently, Lemma 2 applied with $\Phi=\exp$ and $L=1$ and combined with the second inequality in (1) (or, more precisely, the upper estimate on $N^{\prime \prime}$ given by Lemma 1) yields the upper estimate for $N(G, \varepsilon)$. The lower estimate is obtained similarly by applying Lemma 2 to $\Phi=\exp ^{-1}, L=2.5$ and $K=\{u \in G: \rho(u, I) \leq \pi / 4\}$, using the first inequality in (1) (or, again more precisely, the lower estimate on $\tilde{N}$ given by Lemma 1), the last assertion of Lemma 4 and Proposition 6(a). (Proposition 6(a) is needed only for the definition of $K$ and its use may be avoided here.)

If $M \neq G$, the approach will be similar, but the situation is (necessarily) more complicated. Let $q: G \rightarrow G / H=M$ be the quotient map and consider the short exact 
sequence $0 \rightarrow H \rightarrow G \rightarrow M \rightarrow 0$ and the induced sequence of maps between the tangent spaces (at resp. $I \in H, I \in G$ and $H \in M=G / H$ )

$$
0 \rightarrow \mathcal{H} \rightarrow \mathcal{G} \rightarrow T_{H} M \rightarrow 0
$$

and so $T_{H} M$ can be identified with the quotient space $\mathcal{G} / \mathcal{H}$; we mean by that isometrically identified whenever all metric structures are induced by a given unitarily invariant norm. Since the derivative of the exponential map at 0 is the identity (in particular an isometry), we can realize that identification by the canonical factorization of the derivative of $q \circ \exp$ at 0 (which maps $\mathcal{G}$ to $T_{H} M$ and vanishes on $\mathcal{H}$ ) through $\mathcal{G} / \mathcal{H}$. This shows that (at least small) neighborhoods in $M$ resemble balls in the normed space $\mathcal{G} / \mathcal{H}$ and gives some heuristic evidence that inequalities of type (2) may hold for $M$. However, for a proof of such an inequality one needs "uniform isomorphic" (rather than "infinitesimal") estimates, and we will obtain these under some additional technical assumptions. Since the additive structure on $\mathcal{G}$ and the group structure on $G$ are not intertwined by the exponential (or any other) map, it will be more convenient to identify $\mathcal{G} / \mathcal{H}$ with $\mathcal{X}=\mathcal{H}^{\perp}$ (the orthogonal complement of $\mathcal{H}$ in $\mathcal{G}$ ) and to consider $\Phi=q \circ \exp _{\mid \mathcal{X}}$, hoping that the direct sum $\mathcal{X} \oplus \mathcal{H}=\mathcal{G}$ is "well-behaving" with respect to the operator norm (or any other unitarily invariant norm that we may need to consider), which happens in many natural examples. This leads to our first invariant related to a homogeneous space. We set

$$
\kappa(M)=\left\|P_{\mathcal{X}}\right\|=\left\|I-P_{\mathcal{H}}\right\|,
$$

where $P_{\mathcal{E}}$ denotes the orthogonal projection from $\mathcal{G}$ onto $\mathcal{E}$ and $\|\cdot\|$ is calculated with respect to the operator norm on $\mathcal{G}$.

Before stating the results, we will present a simple but illuminating example which shows that, in general, the "linearization" of $M$ of the type suggested above may work only on the "infinitesimal" scale (i.e. only very small neighborhoods are "equivalent" to balls in the tangent space), and which leads to one more invariant of $M$. Let $G=U(n)$ and $H=$ $S U(n)$. It is then easily seen that $M=U(n) / S U(n)$ is isometric to a circle of radius $1 / n$ and so covering numbers of $N(M, \varepsilon)$ are "trivial" if $\varepsilon>\pi / n$. (Since $M$ is 1-dimensional, it is necessarily "isotropic" and so there are neighborhoods resembling segments of size comparable to the diameter of $M$; in particular (2) still holds. However, one can also produce "nonisotropic" examples: consider e.g., $H=\{I\} \times S U(n-1) \subset U(n)=G$.) The reason for this phenomenon is that $S U(n)$ (or, via the exponential map, $\mathcal{H}$ ) is very "densely woven" into $U(n)$. For example, $e^{2 \pi i / n} I \in S U(n)$ and $\left\|e^{2 \pi i / n} I-I\right\|<2 \pi / n$ (more precisely, $\rho\left(e^{2 \pi i / n} I, I\right)=2 \pi / n$ by Proposition $6($ a)), even though the shortest path connecting $I$ and $e^{2 \pi i / n} I$ and contained in $S U(n)$ is of length $2 \pi(1-1 / n)$ (this follows e.g. from the proof of Proposition 6(a) in [22], the length in question must be $\geq$ than the length of the shortest path connecting $(-2 \pi+2 \pi / n, 2 \pi / n, \ldots, 2 \pi / n)$ and 0 in $\ell_{\infty}^{n} / S_{n}$ that is contained in the plane $\left\{\left(x_{k}\right) \in \mathbf{R}^{n}: \sum x_{k}=0\right\}$; another way to express this is that $e^{2 \pi i / n} I=e^{x}$ with $x \in \mathcal{H}$ forces $\left.\|x\| \geq 2 \pi(1-1 / n)\right)$. To quantify the phenomenon we introduce the following concept. Given $\theta>0$, we will say that a closed connected Lie subgroup $H$ of $G=U(n)$ (or $S O(n))$ is $\theta$-woven if whenever $u \in H$ satisfies $\rho(u, I) \leq \theta$ ( $\rho$ is the intrinsic metric induced by the operator norm $\left.\|\cdot\|_{\infty}\right)$, then there exists $x \in \mathcal{H},\|x\|_{\infty}<\pi$ such that $u=e^{x}$. If $M=G / H$, we set 


$$
\theta(M)=\sup \{\theta>0: H \text { is } \theta \text {-woven }\}=\operatorname{dist}\left(I, H \backslash \exp \left(B_{\mathcal{H}}(\pi)\right),\right.
$$

the distance being calculated using $\rho$. We then have

TheOREM 8. In the notation above, assume that $\kappa(M)=1$. Then, for any $\varepsilon \in$ $(0, \operatorname{diam} M]$,

$$
N(M, \varepsilon) \leq\left(\frac{C \operatorname{diam} M}{\varepsilon}\right)^{d},
$$

where $d$ is the (real) dimension of $M$, $\operatorname{diam} M$ is calculated with respect to $\rho_{M}$, and $C>0$ is a universal constant. Moreover, if $\varepsilon \in(0, \theta(M) / 4]$, then

$$
N(M, \varepsilon) \geq\left(\frac{c \theta(M)}{\varepsilon}\right)^{d},
$$

where $c>0$ is a universal constant. The last estimate holds also if $\kappa(M)>1$, but the constant $c$ may then depend on $\kappa(M)$.

P r o of. As suggested earlier, the proof will involve applying Lemma 2 to the (properly restricted) map $q \circ \exp _{\mid \mathcal{X}}$ and its inverse, where $\mathcal{X}$ is the orthogonal complement in $\mathcal{G}$ of $\mathcal{H}$ (the Lie subalgebra of $\mathcal{G}$ corresponding to the subgroup $H$ ), and will be based on two lemmas that follow. Given $r>0$, let $B \mathcal{X}(r)$ be the ball in $\mathcal{X}$ of radius $r$ (with respect to the operator norm) and centered at the origin. We then have, in the notation of Theorem 8:

Lemma 9. If $\kappa(M)=1$, then $q\left(\exp \left(B_{\mathcal{X}}(\operatorname{diam} M)\right)\right)=M$.

Lemma 10. There exist positive constants $\lambda=\lambda(\kappa(M))$ and $r_{0}=r_{0}(\kappa(M))$ such that if $r=\min \left\{r_{0}, \theta(M) / 4\right\}$ and $x, x^{\prime} \in B_{\mathcal{X}}(r)$, then

$$
\rho_{M}\left(q\left(e^{x}\right), q\left(e^{x^{\prime}}\right)\right) \geq \lambda\left\|x-x^{\prime}\right\| .
$$

Remarks. (i) Calculating $\theta(M)$ is not difficult, particularly when $H$ is semisimple. Indeed, suppose $\theta(M)<\pi$ (clearly the maximal possible value) and let $u \in H \backslash \exp \left(B_{\mathcal{H}}(\pi)\right.$ ) be such that $u=e^{h},\|h\|_{\infty} \geq \pi$, while $\|u-I\|_{\infty}=\theta(M)<\pi$, in particular $u=e^{x}$ for some $x \in \mathcal{G},\|x\|_{\infty}<\pi$. Since the commutants of $u$ and $x$ are the same, it follows that $\theta(M)$ is "witnessed" inside a torus $\mathcal{T}$ in $G$ containing $u$; moreover, $\mathcal{T}$ may be assumed to contain the one-parameter semigroup $\left\{e^{t x}: t \in \mathbf{R}\right\}$ and to be such that $\mathcal{T} \cap \mathcal{H}$ is maximal in $H$. Consequently, to determine $\theta(M)$ we only need to examine maximal tori in $H$ and their extensions to maximal tori in $G$. This is particularly easy if $H$ is semi-simple: all configurations of the tori in question are then related by conjugation, and since the metric we consider is invariant under conjugation, it suffices to check just one such configuration. Such an examination will also reveal that $\theta(M)=\pi$, should that be the case.

(ii) Since $\operatorname{diam} U(n)=\pi$ (we recall that we are dealing with the intrinsic metric induced by $\|\cdot\|_{o p}$ ) and $q$, being a quotient map, is a contraction, one always has $\operatorname{diam} M \leq$ $\pi$. In any case, by Proposition 6 , one can always calculate $\operatorname{diam} M$ by examining images of one-parameter semigroups of $G$ under $q$.

(iii) While there seems to be no reason for the assertion of Lemma 9 to hold when $\kappa(M)>1$, it is imaginable that we always have, e.g., $q\left(\exp \left(B_{\mathcal{X}}(\kappa(M) \operatorname{diam} M)\right)\right)=M$. However, we haven't found any argument to that (or similar) effect. 
(iv) If $M=G_{n, k}$ (the Grassmann manifold), one verifies directly that $\kappa(M)=1$ and $\operatorname{diam} M=\pi / 2$. The former follows from the fact that $\mathcal{X}$ consists of those matrices in $\mathcal{G}$ $(=u(n)$ or $s o(n))$ that are of the form (cf. (4))

$$
\left[\begin{array}{cc}
0 & x \\
-x^{*} & 0
\end{array}\right]
$$

The latter is elementary: for two ( $k$-dimensional) subspaces $E, F$ of $\mathbf{R}^{n}$ (resp. $\left.\mathbf{C}^{n}\right)$ ), $\rho_{G_{n, k}}(E, F)$ is the largest of the main angles between $E$ and $F$ (see (4) and comments following it for the framework and, e.g., [17], p. 174 for the more precise analysis). Finally, it follows immediately from Remark (ii) above that $\theta\left(G_{n, k}\right)=\pi$ (the maximal tori in $H$ are also maximal in $G$ ).

(v) If $\kappa(M)=1$, one can take $r=0.12$ and $\lambda=0.4$ in Lemma 10. If, moreover, $x^{\prime}=0$, one can take $r=5 / 9$ and $\lambda=0.4$; it follows that $q \circ \exp \left(B_{\mathcal{X}}(5 / 9)\right) \supset\{E \in G / H$ : $\left.\rho_{M}(E, q(I)) \leq 2 / 9\right\}$ and that $q \circ \exp ^{-1}$ restricted to any of these two sets is Lipschitz with constant 4.5 .

(vi) The proof gives $\lambda(t)$ and $r_{0}(t)$ to be of order $1 / t$. The argument would be slightly more efficient if we considered $\mathcal{X}$ as endowed with the quotient norm $\mathcal{G} / \mathcal{H}$, which is more natural in the context.

Assuming the two lemmas above, Theorem 8 is shown almost exactly as Theorem 7: one applies Lemma 2 , first with $\Phi=q \circ \exp _{\mid \mathcal{X}}, L=1$ and $K=B_{\mathcal{X}}(\operatorname{diam} M)$ for the upper estimate and then with the inverse map restricted to $K=q\left(\exp \left(B_{\mathcal{X}}(r)\right)\right)$ and with $L=\lambda^{-1}$ for the lower estimate. (All the fine points are hidden in Lemmas 9 and 10.)

Proof of Lemma 9. We will show that, for every $p \in[2, \infty)$, setting

$$
K_{p}=\left\{x \in \mathcal{X}:\|x\|_{p} \leq n^{1 / p} \operatorname{diam} M\right\}
$$

(i.e. $K_{p}$ is a ball in $\mathcal{X}$ of radius $n^{1 / p} \operatorname{diam} M$ in the Schatten $C_{p}$-norm $\|\cdot\|_{p}$ ), we have

$$
q\left(\exp \left(K_{p}\right)\right)=M
$$

the assertion of the Lemma will then follow by letting $p \rightarrow \infty$. To this end, observe that since the $\mathbf{R}$-linear orthogonal projection $M(n) \rightarrow \mathcal{G}$ is of norm one (with respect to any unitarily invariant norm), $\kappa(M)$ equals the norm of the orthogonal projection from $M(n)$ onto $\mathcal{X}$. Now, since $\left(M(n),\|\cdot\|_{p}\right)$ is (for $p \geq 2$ ) a complex interpolation space between $\left(M(n),\|\cdot\|_{\infty}\right)$ and $\left(M(n),\|\cdot\|_{2}\right)$, it follows that $P_{\mathcal{X}}$ is also contractive with respect to the $C_{p}$-norm (for any $p \geq 1$; more generally, of norm $\leq \kappa(M)^{|1-2 / p|}$ ). Furthermore, since the $C_{p}$-norm is strictly convex for $p \in(1, \infty)$, we conclude that

$$
y \notin \mathcal{X} \Rightarrow\left\|y-P_{\mathcal{H}} y\right\|_{p}=\left\|P_{\mathcal{X}} y\right\|_{p}<\|y\|_{p} .
$$

For clarity, we will denote by $M_{p}$ the manifold $M$ equipped with the quotient metric $\rho_{p, M}$ induced by the Schatten $C_{p}$-norm. Note that since the operator norm and the $C_{p}$-norm differ by a factor $n^{1 / p}$ at the most, we have $\operatorname{diam} M_{p} \leq n^{1 / p} \operatorname{diam} M$. Let $g H \in M_{p}$ and let $\gamma$ be the shortest geodesic in $M_{p}$ connecting $H$ and $g H$, then $\ell(\gamma) \leq n^{1 / p} \operatorname{diam} M$. Let $\tilde{\gamma}$ be a transversal lifting of $\gamma$ to $G$, i.e. a curve in $G$ such that $q \circ \tilde{\gamma}=\gamma$ and $\ell(\tilde{\gamma})=\ell(\gamma)$. Then of course $\tilde{\gamma}$ is a geodesic in $G$ (with respect to the intrinsic metric $\rho_{p}$ induced by the $C_{p}$-norm) and without loss of generality we may assume that the initial point of $\tilde{\gamma}$ 
is $I$. By Proposition 6, $\tilde{\gamma}$ must be (perhaps after a change of parameter) of the form $\tilde{\gamma}(t)=e^{t y}, 0 \leq t \leq 1$ for some $y \in \mathcal{G}$ and $\ell(\tilde{\gamma})=\|y\|_{p} \leq n^{1 / p} \operatorname{diam} M$, and so (12) will follow if we show that $y \in \mathcal{X}$. Indeed, if that were not the case, (13) would imply that $\left\|y-P_{\mathcal{H}} y\right\|_{p}<\|y\|_{p}$ and so, for $t>0$ sufficiently small we would have

$$
\rho_{p, M}\left(e^{t y} H, H\right) \leq \rho_{p}\left(e^{t y}, e^{t P_{\mathcal{H}} y}\right) \leq\left\|t y-t P_{\mathcal{H}} y\right\|_{p}<t\|y\|_{p}
$$

and consequently

$$
\begin{aligned}
\ell(\tilde{\gamma}) & =\rho_{p, M}(H, g H)=\rho_{p, M}\left(H, e^{y} H\right) \\
& \leq \rho_{p, M}\left(H, e^{t y} H\right)+\rho_{p, M}\left(e^{t y} H, e^{y} H\right)<t\|y\|_{p}+(1-t)\|y\|_{p},
\end{aligned}
$$

a contradiction. This proves Lemma 9.

Proof of Lemma 10. We need to show that if $x, x^{\prime} \in B_{\mathcal{X}}(r)$ and $h \in \mathcal{H}$, then

$$
\Delta \equiv \rho\left(e^{x^{\prime}}, e^{x} e^{h}\right) \geq \lambda\left\|x-x^{\prime}\right\| .
$$

Since $\left\|x-x^{\prime}\right\| \geq \rho\left(e^{x^{\prime}}, e^{x}\right)=\rho\left(e^{-x} e^{x^{\prime}}, I\right)$ and $\Delta=\rho\left(e^{-x} e^{x^{\prime}}, e^{h}\right)$, it is enough to consider $h \in \mathcal{H}$ such that

$$
\rho\left(e^{h}, I\right) \leq(1+\lambda)\left\|x-x^{\prime}\right\| \leq(1+\lambda) 2 r \leq 4 r .
$$

If $r \leq \theta(M) / 4$ (or just $2(1+\lambda) r \leq \theta(M)$ ), it follows from the definition of $\theta(M)$ (i.e. (11)) that $h \in \mathcal{H}$ may be further assumed to satisfy $\|h\|_{\infty}<\pi$, hence

$$
\|h\|_{\infty}=\rho\left(e^{h}, I\right) \leq(1+\lambda)\left\|x-x^{\prime}\right\| \leq(1+\lambda) 2 r \leq 4 r .
$$

Now, by Lemma 4 and Lemma 5 ,

$$
\begin{aligned}
\Delta & \equiv \rho\left(e^{x^{\prime}}, e^{x} e^{h}\right) \geq \rho\left(e^{x^{\prime}} e^{-h / 2}, e^{x} e^{h / 2}\right) \\
& \geq \rho\left(e^{x^{\prime}-h / 2}, e^{x+h / 2}\right)-\left\|\left[x^{\prime}, h / 2\right]\right\|-\|[x, h / 2]\| \\
& \geq \phi(r+\|h\| / 2)\left\|x-x^{\prime}-h\right\|-2 r\|h\| \\
& \geq \phi(r+\|h\| / 2)\left\|x-x^{\prime}\right\|-2 r\|h\| \\
& \geq(\phi(r+(1+\lambda) r)-2 r(1+\lambda))\left\|x-x^{\prime}\right\| \\
& \geq(\phi(3 r)-4 r)\left\|x-x^{\prime}\right\|,
\end{aligned}
$$

where $\phi(\cdot)$ is the function from Lemma 4 . It is now clear from Lemma 4 that if $r>0$ is small enough, then $\phi(3 r)-4 r>0$. A more careful calculation along the same lines shows that if $r=.12$, then $\lambda=.4$ works (as indicated in Remark (v) above).

Finally, if $\kappa(M)>1$, we can only use $\left\|x-x^{\prime}-h\right\| \geq \kappa(M)^{-1}\left\|x-x^{\prime}\right\|$ in the fourth inequality in the above argument. This leads to the minorization $\left(\phi(3 r) \kappa(M)^{-1}-4 r\right) \| x-$ $x^{\prime} \|$ for $\Delta$ and to $r_{0}$ and $\lambda$ of order $\kappa(M)^{-1}$ in the assertion of the Lemma.

4. Extensions and other tricks. The scheme presented in the preceding section yields reasonable estimates for covering numbers $N(M, \varepsilon)$ (with respect to the metric induced by the operator norm) of a homogeneous space $M=G / M$ whenever $\varepsilon \leq \operatorname{diam} M$ or $\varepsilon \leq \theta(M)$ (for the upper and lower estimate respectively) and whenever $\kappa(M)$ is "under control". This leaves several cases and gray areas that are not covered.

(i) The range $\theta(M)<\varepsilon<\operatorname{diam} M$ even if $\kappa(M)=1$ and, in general, a clarification of the role of the ratio diam $M / \theta(M)$ (the lower and upper estimates differing roughly by $\left.(\operatorname{diam} M / \theta(M))^{d}\right)$.

(ii) The upper estimate whenever $\kappa(M)>1$, but still "under control". 
(iii) The case when we do not control $\kappa(M)$.

With regard to (i), a modification of the example that led to the definition of $\theta(M)$ suggested there $(H=\{I\} \times S U(n-1) \subset U(n)=G)$ shows that it is possible for diam $M$ and $\theta(M)$ to differ by a large factor (of order $n$ in that case). Even though an analysis of such cases is imaginably possible, it would be clearly combinatorial and/or algebraic in nature and we do not attempt it here.

Concerning (iii), it is also conceivable that the phenomenon of having $\kappa(M)$ "large" can be "dissected" and expressed in terms of combinatorial/algebraic invariants suggested above, but, again, in the examples motivating this work (see below) we have $\left\|P_{\mathcal{H}}\right\|=1$ and hence $\kappa(M) \leq 2$.

It remains to analyze the gap related to (ii): the examples with, say, $1<\kappa(M) \leq 2$ do naturally occur and it would be nice to have, at least for that case, an upper estimate for covering numbers of $M$ of the type $(C \operatorname{diam} M / \varepsilon)^{d}$ (cf. also Remark (iii) following Lemma 10). Unfortunately, we do not know how to settle that question in full generality. Instead, we present a "bag of tricks" that allow to handle various special cases. This, and some comments concerning covering numbers relative to metrics generated by unitarily invariant norms other than the operator norm constitutes this section.

The first observation is that trying to mimic the proof of Lemma 9 in the case when $\kappa(M)>1$ one arrives at the following picture. Let $Q: \mathcal{G} \rightarrow \mathcal{G} / \mathcal{H}$ be the quotient map, and consider the semi-norm $p$ on $\mathcal{G}$ defined by $p(x)=\|Q x\|_{\infty}$. Let $\Lambda: \mathcal{G} / \mathcal{H} \rightarrow \mathcal{G}$ be a norm-preserving lifting of $Q$ (in general nonlinear). The argument imitating the proof of Lemma 9 connects then geodesics in $M$ with "rays" in the range of $\Lambda$ and we could give upper estimates for entropy of $M$ if we were able to control entropy of the range of $M$ (e.g. with respect to the semi-norm $p$ ).

The two specific subgroups of $U(n)$, for which estimates for covering numbers of the respective homogeneous spaces are of interest from the point of view of free probability (cf. [25], Remark 7.2 and [26]), consist of unitaries of some $C^{*}$-subalgebras of $M(n)$, namely

(I) The "block-diagonal" algebra: the commutant of $\left\{P_{1}, P_{2}, \ldots, P_{m}\right\}$, where $P_{j}$ 's are orthogonal projections whose ranges form an orthogonal decomposition of $\mathbf{C}^{n}$; the corresponding homogeneous space is then a (partial) flag manifold.

(II) The "tensor-factor" algebra: if $n=m k$, identify $\mathbf{C}^{n}$ with $\mathbf{C}^{m} \otimes \mathbf{C}^{k}$ and consider matrices of the form $I \otimes x, x \in M(k)$; these can be also thought of as block matrices with $m$ identical $k \times k$ blocks along the diagonal.

In both cases (I) and (II) the subgroup $H$ (resp. the Lie algebra $\mathcal{H}$ ) consists of (all) unitaries (resp. skew-symmetric matrices) having form (I) or (II), and the conditional expectation is a norm one (with respect to any unitarily invariant norm) projection from $\mathcal{G}$, the Lie algebra of $G=U(n)$, onto $\mathcal{H}$, in particular $\kappa(G / H) \leq 2$. However, except for the case $m=1$ or $m=2$ (the Grassmann manifold for $(\mathrm{I})$ ), we have $\kappa(G / H)>1$.

Concerning the other parameters, it is easily seen (cf. Remarks (i) and (ii) following Lemma 10) that in all cases $\theta(G / H)=2$ and $\pi / 2 \leq \operatorname{diam} G / H \leq \pi$. Accordingly, Theorem 8 gives good lower estimates for the covering numbers of $G / H$ and it remains to handle the upper ones. We will use the following (ad hoc) 
Theorem 11. Let $\alpha \in(0,1 / 2]$; let $n, G(=U(n)$ or $S O(n)), H, M=G / H, \mathcal{G}, \mathcal{H}$, and $d$ be as before and assume that

$$
\min \left\{\theta(M), \operatorname{diam} M, \kappa(M)^{-1}\right\} \geq \alpha .
$$

Furthermore, assume that one of the following holds:

(a) $\operatorname{dim} H \leq(1-\alpha) \operatorname{dim} G$,

(b) $H$ acts reducibly on $\mathbf{C}^{n}$ (resp. $\mathbf{R}^{n}$ ) and there is a reducing subspace $E$ with $\alpha n \leq$ $\operatorname{dim} E \leq(1-\alpha) n$,

(c) $H$ acts reducibly on $\mathbf{C}^{n}$ (resp. $\mathbf{R}^{n}$ ) and there is a reducing subspace $E$ with $\operatorname{dim} E \equiv k \geq \alpha$ and such that the orthogonal decomposition $\mathbf{C}^{n}=E \oplus E^{\perp}$ induces an isomorphism $H \rightarrow U(k) \times H_{0}$ for some subgroup $H_{0}$ of $U(n-k)\left(r e s p . \mathbf{R}^{n}, S O(k), S O(n-k)\right)$.

Then, for any $\varepsilon \in(0, \operatorname{diam} M]$,

$$
(c / \varepsilon)^{d} \leq N(M, \varepsilon) \leq(C / \varepsilon)^{d},
$$

where $c, C>0$ are constants depending only on $\alpha$.

COROllary 12. If $H \subset U(n)$ is the group of unitaries of a "block-diagonal" or "tensor-factor" algebra (described in $(I)$ or $(I I)$ ), then the assertion of Theorem 11 holds (e.g., with constants corresponding to $\alpha=1 / 3)$.

P r o of. We may of course assume $m \geq 2$. In the case (II) the condition (b) of Theorem 11 is always satisfied (with $\alpha=1 / 3$ ). The same is true in the case (I) except if one of the projections $P_{j}$ is of rank $\geq n / 3$, in which case (c) holds.

Remarks. (i) As was pointed out in [25], Remark 7.2, the estimates for covering numbers given by our Corollary 12 (the "block-diagonal" case) allow sharp free entropy and free entropy dimension estimates. Similarly, the "tensor-factor" case of the Corollary implies estimates for free entropy and free entropy dimension of certain generators of free product von Neumann algebras ([26]).

(ii) The "block-diagonal" case of Corollary 12 implies estimates for covering numbers of some sets of matrices needed in [6].

Proof of Theorem 11. As observed earlier, it is enough to show the upper estimate.

(a) The condition (a) is equivalent to $\operatorname{dim} M \geq \alpha \operatorname{dim} G$. It follows from Theorem 7 that, for any $\varepsilon \in(0,2], G$ (hence, by Lemma $2, M=G / H)$ admits an $\varepsilon$-net of cardinality $\leq(C / \varepsilon)^{\operatorname{dim} G}$. If $\varepsilon \geq \beta$ and (a) holds, this does not exceed $C(\alpha, \beta)^{\operatorname{dim} M}$ (the real dimension). It follows now from Lemma 10 that the image of $B_{\mathcal{X}}(r)$ (where $r=r(\alpha)$ ) contains a ball in $M$ of radius $r_{1}=r_{1}(\alpha)$, and so the former and the latter admit, for $\varepsilon \leq r$, an $\varepsilon$-net of cardinality $\leq\left(C_{1} r / \varepsilon\right)^{\operatorname{dim} M}$. Combining this with the preceding observation (applied to $\beta=r_{1}$ ) we get the required upper estimate.

(b) The condition (b) implies (a) (with $\alpha(1-\alpha)$ in place of $\alpha$ ).

(c) Since the arguments in the real and complex case are identical, we restrict the discussion to the latter. The condition (c) is not included in (b) only if $\operatorname{dim} E \equiv k>$ $(1-\alpha) n$, in particular $k>n / 2$. Let $H_{1} \subset G$ be the subgroup of the form $U(k) \times\{I\}$ in the sense indicated in the condition (c), then $H_{1} \subset H$ and so $M=G / H$ is a quotient of $M_{1}=G / H_{1}$. Now $M_{1}$ is isomorphic to $G_{n, k} \times U(n-k)$ (for sure Lipschitz isomorphic 
with constant 2 if the product metric on the latter is defined in the " $\ell_{\infty}$ sense"), and so, by Theorems 7 and 8 , it admits, for any $\varepsilon \in(0,2]$, an $\varepsilon$-net of cardinality $\leq\left(\frac{C_{2}}{\varepsilon}\right)^{\operatorname{dim} M_{1}}$ (again, the real dimension). Since $k>n / 2$ implies $\operatorname{dim} M_{1}<2 \operatorname{dim} M$, arguing as in (a) we obtain the assertion.

In some applications (see e.g. [19], [20]) it is important to know the metric entropy of $M$ equipped with a metric induced by unitarily invariant norms other than the operator norm. The scheme presented in this paper can be adapted to yield fairly sharp results in the general case. Indeed, Lemmas 4, 5 and 6 involve statements about generic unitarily invariant norms. Similarly, Lemma 10 and its proof carry over almost word by word to the case of an arbitrary unitarily invariant norm $\|\cdot\|$ once the parameters such as $\theta$ and $\kappa$ are properly interpreted: the balls $B_{\mathcal{H}}(\cdot), B_{\mathcal{X}}(\cdot)$ are to remain to be defined by the operator norm $\|\cdot\|_{\infty}$, but $\theta(M)$ has to be the distance between $I$ and $H \backslash \exp \left(B_{\mathcal{H}}(\pi)\right)$ in the intrinsic metric on $G$ induced by $\|\cdot\| ; \kappa(M)$ may be calculated using $\|\cdot\|$ (which results in a quantity not larger than the one given by the operator norm, in particular $\kappa(M)=1$ if $\|\cdot\|$ is the Hilbert Schmidt norm). The "linearization" procedure can be then implemented and the problem is reduced to estimating covering numbers of balls in $\mathcal{X}$ in the operator norm with respect to $\|\cdot\|$. As indicated in section 2 (see Lemma 1 and the paragraph preceding it), there exist numerous tools for obtaining such estimates. In particular, in many natural cases (e.g. $M=U(n), S O(n)$ or the Grassmann manifolds $\left.G_{n, k}\right)$, the volumes of a ball in $X$ with respect to a unitarily invariant norm $\|\cdot\|$ and the inscribed operator norm ball differ by a factor $C^{d}, C$ - a universal constant (this is easily implied e.g., by classical facts from [16], [5], cf. [18], p. 162; see also [9] or [15]), which allows to use Lemma 1 to show that $\log N(M, \varepsilon) \approx d \log (\operatorname{diam} M / \varepsilon)$ (and $\operatorname{diam} M \approx\|I\|$ if $M=U(n)$ or $S O(n), \operatorname{diam} M \approx\|P\|$, where $P$ is an orthogonal projection of rank equal to $\min \{k, n-k\}$ if $\left.M=G_{n, k}\right)$; these cases have been worked out in [17].

\section{References}

[1] K. Ball, E. A. Carlen and E. H. Lieb, Sharp uniform convexity and smoothness inequalities for trace norms, Invent. Math. 115 (1994), no. 3, 463-482.

[2] R. Bhatia, Perturbation bounds for matrix eigenvalues, Pitman Research Notes 162, Longman Scientific \& Technical, Harlow 1987.

[3] H. Busemann, Metric methods in Finsler spaces and the foundations of geometry, Annals Math. Studies 8, Princeton University Press, Princeton, 1942.

[4] B. Carl and I. Stephani, Entropy, Compactness and Approximation of Operators, Cambridge University Press, Cambridge, 1990.

[5] S. Chevet, Séries de variables aléatoires gaussiennes à valeurs dans $E \hat{\otimes}_{\varepsilon} F$, Séminaire sur la géométrie des espaces de Banach 1977-78, Ecole Polytechnique, Palaiseau.

[6] K. Dykema, personal communication.

[7] L. Ge, Prime factors, Proc. Natl. Acad. Sci. USA 93 (1996), 12762-12763.

[8] L. Ge, Applications of free entropy to finite von Neumann algebras, Amer. J. Math. 119 (1997), 467-485

[9] E. D. Gluskin, The diameter of the Minkowski compactum is roughly equal to $n$, Functional Anal. Appl. 15 (1981), 72-73. 
[10] I. C. Gohberg and M. G. Krein, Introduction to the Theory of Linear Nonselfadjoint Operators, Nauka, Moscow 1965. English transl.: AMS 1969.

[11] S. Helgason, Differential Geometry, Lie Groups and Symmetric Spaces, Academic Press, New York 1978.

[12] D. Herrero and S. J. Szarek, How well can an $n \times n$ matrix be approximated by reducible ones?, Duke Math. J. 53 (1986), 233-248.

[13] A. Pajor, Metric entropy of the Grassmann manifold, Proceedings of the MSRI Convex Geometry Semester (Spring 1996), to appear.

[14] G. Pisier, The Volume of Convex Bodies and Banach Space Geometry, Cambridge University Press, Cambridge 1989.

[15] J. Saint-Raymond, Sur le volume des idéaux d'opérateurs classiques, Studia Math. 80 (1984), 63-75.

[16] L. A. Santaló, Un invariante afin para los cuerpos convexos del espacio de $n$ dimensiones, Port. Math. 8(1949), 155-161.

[17] S. J. Szarek, Nets of Grassmann manifolds and orthogonal groups, Proceedings of Banach Space Workshop, University of Iowa Press 1982, 169-185.

[18] S. J. Szarek, The finite dimensional basis problem with an appendix on nets of Grassmann manifolds, Acta Math. 151 (1983), 153-179.

[19] S. J. Szarek, An exotic quasidiagonal operator, J. Funct. Anal. 89 (1990), 274-290.

[20] S. J. Szarek, Spaces with large distance to $l_{\infty}^{n}$ and random matrices, Amer. J. Math. 112 (1990), 899-942.

[21] S. J. Szarek, Metric entropy of homogeneous spaces and Finsler geometry of classical Lie groups, MSRI preprint 1997-010.

[22] S. J. Szarek, Geodesics for Invariant Finsler Geometries on Classical Lie Groups, to appear.

[23] N. Tomczak-Jaegermann, Banach-Mazur distances and finite-dimensional operator ideals, Longman Scientific \& Technical, Harlow 1989.

[24] V. Varadarajan, Lie Groups, Lie Algebras and Their Representations, Springer Verlag, New York 1984.

[25] D. Voiculescu, The analogues of entropy and of Fisher's information measure in free probability theory. III. The absence of Cartan subalgebras, Geom. Funct. Anal. 6 (1996), 172-199.

[26] D. Voiculescu, personal communication. 